\title{
Parole médiatique en temps de crise
}

Étude de cas

Mediatic reports in time of crisis.

\section{Simone Bonnafous}

\section{(2) OpenEdition \\ Journals}

Édition électronique

URL : http://journals.openedition.org/edc/2687

DOI : $10.4000 /$ edc. 2687

ISSN : 2101-0366

Éditeur

Université Lille-3

\section{Édition imprimée}

Date de publication : 1 mai 1994

Pagination : 113-130

ISSN : $1270-6841$

\section{Référence électronique}

Simone Bonnafous, "Parole médiatique en temps de crise », Études de communication [En ligne], 15 | 1994, mis en ligne le 29 décembre 2011, consulté le 01 mai 2019. URL : http:// journals.openedition.org/edc/2687; DOI : 10.4000/edc.2687

Ce document a été généré automatiquement le 1 mai 2019.

(c) Tous droits réservés 


\section{Parole médiatique en temps de crise}

\section{Étude de cas}

Mediatic reports in time of crisis.

\section{Simone Bonnafous}

1 Comment une société « dit-elle » sa propre crise ? Comment raconte-t-elle les processus d'exclusion et de domination, dans l'environnement médiatisé qui est le nôtre?

2 Cette question nous semble aujourd'hui fondamentale à cause de l'ampleur et de la durée de la crise que vivent les sociétés occidentales depuis 20 ans et en même temps du développement sans précédent des médias depuis le début des années 80 . Flot d'images, flot de papiers, flot de mots caractérisent une époque qui ne peut jouer le silence ni l'occultation de ses propres difficultés.

3 Pour expliquer que la montée régulière des exclusions et des inégalités ne provoque pas d'explosion sociale majeure dans un pays comme la France, diverses raisons ont été évoquées par les sociologues et les politistes : effondrement des idéologies alternatives, affaiblissement des organisations politiques et syndicales, permanence d'un système étatique d'assistance et de soutien aux plus démunis, etc... Tous ces motifs - et la liste n'est pas exhaustive - sont sans doute valides; nous voudrions quant à nous, nous interroger sur le rôle que jouent les médias. Non point pour isoler ou diaboliser ces médias, mais parce qu'ils nous paraissent indissociables de notre démocratie, dans ce qu'elle a de meilleur et de pire ${ }^{1}$. De nombreux travaux ont déjà été consacrés aux diverses modalités de «divertissement " médiatiques: narrativation des événements, starisation, dramatisation, spectacularisation, etc... ${ }^{2}$. Dans cet article, nous souhaitons nous interroger sur un autre aspect du fonctionnement des médias, moins étudié parce que peut-être moins typique et moins visible, celui de la distribution et de la hiérarchisation de la parole.

4 Notre méthode est celle de l'analyse de corpus médiatiques. Nous avons choisi de regarder dans le détail comment sont dits « la crise » et «le chômage» dans un journal télévisé (JT) de France 3 d'une part et dans Le Monde de l'autre. Ces deux corpus n'ont pas vocation à représenter toutes les pages du Monde ni toutes les éditions de Soir 3 et encore 
moins toutes les émissions ou tous les journaux d'information. Constituant des moments de focalisation journalistique sur " la crise» et " le chômage», ils nous permettront d'analyser deux systèmes d'organisation discursive différents et de lancer des hypothèses que d'autres analyses de corpus permettront d'enrichir et de nuancer.

5 Se situant explicitement dans le courant d'analyse dit " critique ${ }^{3}$, notre travail prolongera des ouvrages récents qui ont en commun de ne pas négliger « contenu » et « discours » au profit des seules techniques de mise en scène et de ne pas dissocier l'analyse des médias de celle des idéologies. ${ }^{4}$

\section{Soir 3 du 1er juin 1993 - « La crise » des « élites ».}

6 Le 1er juin 1993, le sommet franco-allemand qui réunit à Beaune Helmut Kohl et François Mitterrand est l'occasion pour Christine Ockrent de consacrer le dossier du journal de 22 h $50{ }^{5}$ à la crise économique allemande, et, au delà, européenne. Ce journal nous a paru être un modèle du genre. Non qu'il soit représentatif au sens statistique du terme, mais parce qu'il condense et accentue tous les traits de ce que Jean-G. Padioleau appelle « la rhétorique journalistique ${ }^{6}$ et Gérard Leblanc la philosophie implicite de l'information télévisée ${ }^{7}$.

7 Trois caractéristiques ont retenu notre attention:

- un régime énonciatif monologique, sous des apparences dialogiques,

- un « amortissement » du social et de ses contradictions par l'économique,

- une représentation consensuelle de « la crise » et de ses solutions.

8 Les linguistes ont souvent dénoncé l'illusion du Sujet Parlant maître de son discours et, de Mikhail Bakhtine à Jacqueline Authier ${ }^{8}$, ont traqué toutes les traces des « autres " parlant en Soi : autres " sociaux ", autres de la Loi, autre inconscient... Derrière le monologue, se dévoile ainsi l'hétérogénéité discursive.

9 La conséquence de cette intrication des voix peut être son envers, c'est-à-dire l'homogénéisation discursive - ou ce que Patrick Champagne appelle la « ventriloquie "9. Les réponses des « Français » aux sondages sont l'écho des propos des hommes politiques et des médias, qui sont eux-mêmes en situation d'influence réciproque. Les médias étant devenus le lieu principal de débat et d'information, c'est à une relative uniformisation de la parole publique qu'on assiste au bout du compte.

10 C'est exactement ce qui se passe dans le Soir 3 du 1er juin 1993 où Christine Ockrent orchestre une émission à voix multiples qui est en fait un monologue. Cela tient d'abord au statut donné aux deux invités de l'émission: Jean François-Poncet et Hans Frei. Le premier est présenté une fois à l'écran comme sénateur PR du Tarn et Garonne, mais C. Ockrent ne mentionne jamais oralement son appartenance politique et l'introduit comme " le Président de la Commission des Affaires Economiques du Sénat ». Elle en fait un " expert» (Vous êtes l'un des meilleurs connaisseurs français de l'Allemagne) dont les choix politiques et idéologiques, les revenus, le mode de vie, sont totalement effacés. Comme le sont aussi ceux de "Hans Frei " dont le statut de "banquier allemand de Paris, responsable de la Dresdner Bank " ne semble pas pouvoir entraîner de parti pris dans l'appréciation de la situation économique. Son sérieux et sa compétence se mesurent implicitement à sa puissance économique : Hans Frei, bonsoir. Vous êtes banquier. Vous dirigez en France la très puissante Dresdner Bank. 
11 La «crise» est abordée sous la forme d'un « dossier» dont le traitement relève d'une approche en termes de savoirs et de compétences. Nous sommes dans l'ordre du « vrai» et non pas du " choix ». La parole appartient donc aux experts ou aux «analystes », pour reprendre le mot employé par le correspondant allemand de $\mathrm{C}$. Ockrent à propos des personnes qu'il a interviewées en Allemagne. Ce sont, dans l'ordre, Simon Wiesenthal ${ }^{10}$, Guy Courbon, directeur RHW (groupe Bertrand Faure), Alfred Tacke, secrétaire d'Etat à l'économie du Land de Basse Saxe, Alexander Ecckelpasch, analyste économique (DIW Berlin) et différents «analystes» (sic). On le voit, aucune parole de syndicaliste, d'ouvrier ou d'employé dans cette émission. La parole savante est réservée à l'élite politique et économique dont il semble aller de soi, pour C. Ockrent et peut être pour ses auditeurs, qu'ils ne représentent pas des intérêts catégoriels.

12 L'émission prend un tour très «énarchique» où se combinent la globalisation, la personnification et l'effacement des agents sociaux et de parole.

13 La globalisation est un procédé stylistique courant qui consiste à représenter des pays ou des communautés comme des personnes, appréhendables d'un bloc, sans division ni contradiction. Christine Ockrent évoque une Allemagne solide, sûre de sa puissance économique, de sa productivité et de sa cohésion sociale et affirme que tous les pays européens, on l'a vu à Copenhague, à commencer par la France sont aujourd'hui préoccupés par les problèmes de l'immigration.

14 Peuples et nations se confondent dans une représentation personnifiante de l'économie qui nie les divisions économiques et sociales internes au profit d'une appréhension belliciste de l'économie mondiale.

15 - Jean François-Poncet: ... Le vrai problème c'est la montée en puissance de l'Asie. Nous avons connu d'abord le Japon, nous avons connu ensuite ce qu'on appelait les petits tigres - la Corée, Taïwan - et puis nous connaissons maintenant la Chine, la Chine du Sud ...

16 A l'heure des délocalisations et des flux transfrontières de capitaux et de données, la crise que «racontent»C. Ockrent et Jean François-Poncet, se métamorphose ainsi en une histoire de rendez-vous manqués et de trains renversés :

17 C. Ockrent : ... On a un peu l'impression que nous attendons la reprise allemande et que les Allemands à leur tour attendent la reprise américaine qui vraiment n'est pas au rendez-vous.

18 - Jean François-Poncet : Il y a sûrement ce que vous dites. C'est un peu le serpent qui se mord la queue et on attend que l'autre vous entraîne, que l'autre soit la locomotive qui va vous tirer du fossé dans lequel on est.

19 Bien plus qu'une fonction pédagogique (le niveau intellectuel supposé du public ${ }^{11}$ pourrait permettre sans doute un peu plus de complexité), cette simplification métaphorisante a une fonction idéologique cohérente avec l'emploi des «on» et des «nous» et l'effacement des acteurs :

20 - Pierre Freidenheich : Mais aujourd'hui la cogestion donnée en exemple (par qui ? pour qui ?) ${ }^{12}$ devient un handicap (pour qui, pour quoi ?) lorsque tout bascule (sic) et qu'on (qui ?) ne parle plus qu'en terme de rationalisation et baisse drastique des coûts de fabrication.

21 - Christine Ockrent : Et sur le fond bien sûr une crise économique qui est véritablement le lit de toutes les plaies allemandes. On (qui?) ne le comprend pas assez en France tant on (qui?) reste sur l'idée d'une Allemagne toute puissante avec sa monnaie dominatrice, son économie performante, modèle et phare de l'Europe. 
Le social et ses contradictions sont ainsi subsumés dans les formules et les chiffres de l'économie :

- Pierre Freidenheich : Six semaines de vacances, 35 heures hebdomadaires de travail, defréquentes absences maladies, la productivité allemande devient l'une des plus basses d'Europe pour les salaires les plus élevés. Face à cette nécessité de baisser les coûts, les analystes affirment que trop souvent l'industrie allemande établit des records de suppression d'emplois. /.../

- Hans Frei: Il faut bien revenir aujourd'hui dans des forces qui étaient manifestées dans les années 80 et bien regarder aussi ce qui est aujourd'hui le confort trop important. Par exemple en Allemagne on a aujourd'hui le travail hebdomadaire le plus court en Europe, on a les étudiants les plus âgés, on a les retraités les plus jeunes, on a un système social qui est quand même aujourd'hui mis un peu en cause et il faut bien serrer les ceintures aujourd'hui ...

- Jean François-Poncet: Et je crois que ce problème européen est que nous avons un modèle social qui est un modèle très enviable mais un modèle qui n'est plus soutenu par un avantage de productivité tel que nous l'avons eu pendant longtemps.

Le tableau est sans nuance: «modèle social» pour la France et " confort trop important » pour l'Allemagne. Les inégalités de revenus, de qualité de travail et de vie qui se cachent derrière ces expressions définies sont occultées, comme le sont totalement les conditions faites au salariat des " petits tigres ». Contrairement à ce que nous analyserons plus loin du dossier du Monde sur le chômage, le vécu des hommes et des femmes qui "font " l'économie est ainsi gommé. Le chômage n'est pas évoqué dans sa dimension humaine, mais comme une menace future pour «l'ordre» social :

- Alexander Ecchelpasch (analyste économique) : Il est tout à fait imaginable que les problèmes économiques de l'Allemagne se transforment en problèmes sociaux. Il y a aujourd'hui un risque (pour qui ?) d'explosion sociale.

Présentatrice, reporter et invités communient donc dans l'évocation d'un passé de stabilité, d'ordre et d'équilibre qu'ils opposent à un présent de rupture et de déséquilibre ${ }^{13}$.

Dès le chapeau, le décor est planté en quelques phrases de synthèse :

La crise économique assombrit le sommet franco-allemand qui s'est ouvert aujourd'hui à Beaune, en Bourgogne. Une crise qui ébranle l'Allemagne, autrefois modèle de productivité, d'innovation et d'harmonie sociale, aujourd'hui accablée par le déclin de ses industries lourdes, la chute de sa productivité, la montée du chômage et même de l'inflation. La crise allemande, son évolution et ses conséquences pour nous. Oubliés " la bande à Baader» des années 70, les attentats, les assassinats, les recherches et les procès, oubliée l'exploitation des immigrés turcs dénoncée par Günter Wallraff ${ }^{14}$.

Quant aux analyses et aux solutions proposées, elles sont toutes identiques, Jean FrançoisPoncet, Hans Frei et Christine Ockrent ne faisant que se confirmer, se préciser et se compléter mutuellement. Les remèdes envisagés ont nom " rigueur ", " restructurations ", " productivité ", " effort ", mais les formules restent vagues (il faut bien serrer les ceintures aujourd'huije crois qu'elle (['Europe) va être amenée à faire un énorme effort de productivité sur elle-même) et ne précisent pas comment devront se répartir les efforts ou les crans de ceinture.

Le Soir 3 du 1 er juin 1993 consacré à « la crise » représente sans doute la quintessence du discours d'expertise médiatisé, caractérisé par la réification de l'humain en " dossiers ", « problèmes » et "questions ", l'accaparement de la parole par des élites journalistiques, politiques et économiques et la production d'un discours unique et unificateur qui gomme de la représentation de « la crise» tout antagonisme et toute souffrance concrète. 


\section{Le Monde du 7 au 20 mars 1993 enquête sur l'« interminable chômage ".}

Si nous avons choisi d'étudier dans la seconde partie de cet article une enquête du journal Le Monde sur le chômage, publiée entre le 7 et le 20 mars 1993, c'est qu'elle représente un tout autre genre que le dossier du Soir 3 du 1er juin - et que les deux genres s'éclairent mutuellement. Autant en effet Soir 3 se veut concis et synthétique, autant Le Monde, dont le lectorat recouvre à peu près le public du JT de France 3, prend le temps d'éclairer «les multiples aspects de ce dossier angoissant» (sic. 7/3/93 p. 1). Notre objectif est de montrer la solidarité de ces deux formes journalistiques: d'un côté le «dossier» rapide qui n'a pas à s'embarrasser d'anecdotique et de concret et de l'autre la longue promenade journalistique à travers les tours et les contours d'un mal qui apparaît sans issue.

Motivée par l'approche des élections législatives des 21 et 28 mars, l'enquête du Monde comprend Il livraisons que nous présentons ci-après sous forme de tableau (voir pages suivantes). Au sein du genre global de l'enquête, nous avons distingué plusieurs types d'articles, en nous inspirant d'une typologie que nous avons nous-même construite dans une étude précédente ${ }^{15}$ et de celle que suggère Erik Neveu dans un article intitulé " Rubrique(s) politique(s) $»^{16}$. Cette typologie se veut surtout suggestive, vu l'impossibilité dans laquelle nous serions d'opposer radicalement les genres les uns aux autres ${ }^{17}$.

Contrairement au journal de France 3, l'enquête du Monde n'exclut pas la parole des victimes de la crise. Elles apparaissent à travers " la chronique des non-travaux forcés " de Jean-Pierre Dautun et à travers divers reportages qui émaillent l'enquête et agrémentent les propos austères des experts « extérieurs » au journal ou des journalistes spécialisés. Ces témoignages sont parfois poignants, ils n'en sont pas moins « amortis » et comme « digérés » par la forme générale de l'enquête.

Trois éléments concourent principalement à cet effet

d'amortissement :

-la séparation entre propos « narratifs» et propos « politiques ».

- le fatalisme, au sens quasi étymologique du terme, qui se dégage de l'ensemble du dossier et en particulier des interventions des journalistes spécialisés du Monde.

- le flou des propositions formulées par les experts «extérieurs».

Les propos "narratifs " sont le propre des chômeurs ou des " militants de l'insertion " qui racontent leurs expériences, leurs drames, leurs espoirs, leurs déceptions avec force détails concrets. Le reportage du 17 mars sur "le combat quotidien de quatre demandeurs d'emploi contre la lancinante réalité du chômage » en est un excellent exemple. Le chapeau de l'article présente l'ensemble comme un récit. Chaque personnage est « cadré » dans son physique, son intérieur, sa famille. Les phrases choisies par le journaliste sont chargées de sens et d'émotion :

\section{« INTERMINABLE CHÔMAGE »}

\begin{tabular}{|l|l|l|l|l|}
\hline Date & Titres articles & Genre & Auteur & Page \\
\hline
\end{tabular}




\begin{tabular}{|c|c|c|c|c|}
\hline $\begin{array}{l}7 \text { et } 8 \\
\text { mars } \\
1993\end{array}$ & $\begin{array}{l}1 \text { - « Chômage, le scénario } \\
\text { catastrophe " } \\
2 \text { - « Les partenaires sociaux } \\
\text { s'inquiètent de la situation } \\
\text { financière de l'UNEDIC» }\end{array}$ & $\begin{array}{l}\text { Analyse } 1 \\
\text { Article de reprise } 2\end{array}$ & $\begin{array}{l}\text { Alain Lebaube journaliste } \\
\text { du Monde } \\
\text { Alain Lebaube }\end{array}$ & $\begin{array}{l}\text { p. } 1 \\
\text { et } 13 \\
\text { p. } 13\end{array}$ \\
\hline $\begin{array}{l}9 \text { mars } \\
1993\end{array}$ & $\begin{array}{l}\text { "Un entretien avec Jean } \\
\text { Gandois " (P.-D.G. } \\
\text { Picheney) }\end{array}$ & Interview & $\begin{array}{l}\text { Michel Noblecourt } \\
\text { journaliste du Monde }\end{array}$ & p. 2 \\
\hline $\begin{array}{l}10 \\
\text { mars } \\
1993\end{array}$ & $\begin{array}{l}1 \text { - «Le choc des } 3 \text { millions » } \\
2 \text { - «Les enseignements d'une } \\
\text { histoire » } \\
3 \text { - «L'état-service» } \\
4 \text { - «Chroniques des non- } \\
\text { travaux forcés : «transports } \\
\text { en commun»» }\end{array}$ & $\begin{array}{l}\text { Analyse (la peur des } \\
\text { PDG et des cadres } \\
\text { supérieurs) } \\
\text { Expertise } \\
\text { extérieure3 } \\
\text { Expertise extérieure } \\
\text { Billet d'humeur4 }\end{array}$ & $\begin{array}{l}\text { Alain Lebaube } \\
\text { Robert Salais, chercheur } \\
\text { au CNRS } \\
\text { François rernel, } \\
\text { rédacteur en chef du } \\
\text { Banquet } \\
\begin{array}{l}\text { Jean-Pierre Dautun, cadre } \\
\text { au chômage }\end{array}\end{array}$ & $\begin{array}{l}\text { p.1 et } \\
23 \\
\text { p.2 } \\
\text { p. } 2 \\
\text { p.23 }\end{array}$ \\
\hline $\begin{array}{l}11 \\
\text { mars } \\
1993\end{array}$ & $\begin{array}{l}1 \text { - «Cadres à l'encan " } \\
2 \text { - «Chronique des non- } \\
\text { travaux forcés : « bon } \\
\text { courage»» }\end{array}$ & $\begin{array}{l}\text { Reportage (4 ex. de } \\
\text { cadres au chômage) } \\
\text { Billet d'humeur }\end{array}$ & $\begin{array}{l}\text { Jean Menanteau, } \\
\text { journaliste du Monde } \\
\text { Jean-Pierre Dautun }\end{array}$ & $\begin{array}{l}\text { p. } 19 \\
\text { p. } 19\end{array}$ \\
\hline $\begin{array}{l}12 \\
\text { mars } \\
1993\end{array}$ & $\begin{array}{l}1 \text { - " Les militants de } \\
\text { l'insertion » } \\
2 \text { - « Chroniques des non- } \\
\text { travaux forcés : " me voilà } \\
\text { frais »" }\end{array}$ & $\begin{array}{l}\text { Reportage (3 ex de } \\
\text { responsables } \\
\text { d'entreprises } \\
\text { d'insertion) } \\
\text { Billet d'humeur }\end{array}$ & $\begin{array}{l}\text { Marie-Béatrice Baudet } \\
\text { Jean-Pierre Dautun }\end{array}$ & $\begin{array}{l}\text { p. } 15 \\
\text { p. } 15\end{array}$ \\
\hline $\begin{array}{l}13 \\
\text { mars } \\
1993\end{array}$ & $\begin{array}{l}1 \quad \text { - " Le blues des } \\
\text { économistes devant les files } \\
\text { d'attente " } \\
2 \text { - " Chroniques des non- } \\
\text { travaux forcés : " comment } \\
\text { je suis devenu une ressource } \\
\text { humaine»" } \\
3 \quad \text { " L'utopie des } \\
\text { économètres " }\end{array}$ & $\begin{array}{l}\text { Analyse } \\
\text { Billet d'humeur } \\
\text { Article de reprise }\end{array}$ & $\begin{array}{l}\text { Erik lzsaelewicz } \\
\text { Jean-Pierre Dautun } \\
0\end{array}$ & $\begin{array}{l}\text { p.21 } \\
\text { p.21 } \\
\text { p.21 }\end{array}$ \\
\hline $\begin{array}{l}16 \\
\text { mars } \\
1993\end{array}$ & $\begin{array}{l}\text { "Un entretien avec Jean- } \\
\text { Baptiste de Foucault " } \\
\text { (Commissaire au plan) }\end{array}$ & Interview & $\begin{array}{l}\text { J. M. Colombani, } \\
\text { journalise du Monde }\end{array}$ & p.2 \\
\hline
\end{tabular}




\begin{tabular}{|c|c|c|c|c|}
\hline $\begin{array}{l}17 \\
\text { mars } \\
1993\end{array}$ & $\begin{array}{l}1 \text { - « La course contre la } \\
\text { montre» } \\
2 \text { - « Chronique des non- } \\
\text { travaux forcés : « Prélude à } \\
\text { l'après-midi d'un téléphone } \\
» »\end{array}$ & $\begin{array}{l}\text { Reportage (4 ex. de } \\
\text { chômeurs) } \\
\text { Billet d'humeur }\end{array}$ & $\begin{array}{l}\text { D. Le Guilldoux envoyé } \\
\text { spécial du Monde à } \\
\text { Gravelines } \\
\text { Jean-Pierre Dautun }\end{array}$ & $\begin{array}{l}\text { p. } 19 \\
\text { p. } 19\end{array}$ \\
\hline $\begin{array}{l}18 \\
\text { mars } \\
1993\end{array}$ & $\begin{array}{l}1 \text { « Le tonneau des Danaïdes } \\
\text { ( (ex. de la ville de Vierzon) } \\
2 \text { - « Chroniques des non- } \\
\text { travaux forcés : « Au milieu } \\
\text { de la figure»» }\end{array}$ & $\begin{array}{l}\text { Reportage } \\
\text { Billet d'humeur }\end{array}$ & $\begin{array}{l}\text { Annick Cojean, } \\
\text { journaliste du Monde } \\
\text { Jean-Pierre Dautun }\end{array}$ & $\begin{array}{l}\text { p. } 18 \\
\text { p. } 18\end{array}$ \\
\hline $\begin{array}{l}19 \\
\text { mars } \\
1993\end{array}$ & « Tous étaient touchés» & Analyse & $\begin{array}{l}4 \text { correspondants du } \\
\text { Monde en Grande- } \\
\text { Bretagne, Italie, } \\
\text { Allemagne, Espagne }\end{array}$ & p. 17 \\
\hline $\begin{array}{l}20 \\
\text { mars } \\
1993\end{array}$ & 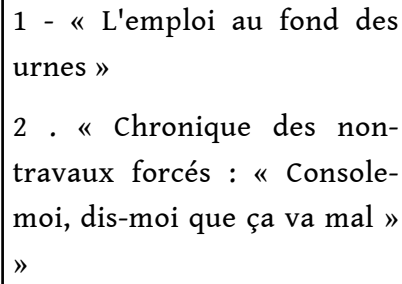 & $\begin{array}{l}\text { Analyse } \\
\text { Billet d'humeur }\end{array}$ & $\begin{array}{l}\text { Michel Noblecourt } \\
\text { Jean-Pierre Dautun }\end{array}$ & $\begin{array}{l}\text { p. } 1 \\
\text { et } 9 \\
\text { p. } 19\end{array}$ \\
\hline
\end{tabular}

1 «L'analyse » est un article assez long, rédigé par un membre du journal présentant une argumentation et dépassant le cadre évémentiel court pour se situer au niveau des principes et des problèmes structurels » (Bonnafous. op. cit. 278). « A la différence de l'éditorial, il n'exprime pas nécessairement la position collective de l'organe qui le publie» (Voyenne, Glossaire des termes de presse, Paris, CFPJ, 1967).

312 « L'« article de reprise », plus long qu'une simple « brève » mêle des propos rapportés et du discours à la 3e personne, ou n'est constitué que de propos rapportés. Souvent produit à partir d'une ou plusieurs dépêches d'agence » (Bonnafous, ibidem).

323 Les « expertises extérieures » peuvent être dénommées « Débats », « Idées ». « Rebonds »), " Point de vue », « Opinions » etc. Elles sont produites par des «chercheurs, consultants, universitaires, sondeurs, intellectuels médiatiques» (Erik Neveu, op. cit.).

334 Le « billet d'humeur » exprime, sous une forme très personnalisée, l'opinion d'un journaliste ou d'une personne extérieure au journal. Parfois constitué en rubrique régulière, il est souvent marqué d'une tonalité affective ou humoristique. (Bonnafous, op. cit. p. 278).

- « Roberto gagne $2200 \mathrm{~F}$ par mois, des allocations de fin de droits. « Ma femme travaille à temps partiel dans un supermarché. Elle touche entre $2000 \mathrm{~F}$ et $3000 \mathrm{~F}$ cela dépend des mois. On vit à calculer. Faut éviter de le montrer au gosse, il est au lycée. Lui, il mange de la viande et nous, des patates. J'ai refusé des bons d'aide alimentaire de la mairie. J'ai ma fierté, on n'est pas des mendiants. Je prie le bon Dieu et ça ne sert à rien, ça aide à réfléchir, peut-être. J'ai tellement de choses dans la tête. Ma vie, c'est simple: c'est pas une vie », dit-il ».

- « Marie-Christine redoute les retards des ASSEDIC, «quelques jours seulement et 
c'est dramatique, on se met à compter au centime près ». Elle, qui touche pour l'instant $4700 \mathrm{~F}$ d'indemnité chômage, angoisse à l'idée de se retrouver « au mois de mai prochain avec $17 \%$ en moins et, quatre mois plus tard, $17 \%$ encore en moins, c'est affolant cette course contre la montre ».».

De même les « chroniques des non-travaux forcés » de Jean-Pierre Dautun ne peuventelles laisser indifférent. Extrêmement travaillées, émaillées de références culturelles, de figures de style, de jeux de mots, elles parviennent à faire pénètrer le lecteur dans la conscience de ce chômeur qui égrène les jours comme un prisonnier. Rédigées à la 1ère personne, sans la médiation du journaliste, elles permettent une identification et une compassion maximum - que facilite sans doute encore la proximité culturelle et sociologique du rédacteur avec les lecteurs du Monde.

- «Prélude à l'après-midi d'un téléphone

Je vais les appeler. Maintenant. Voyons, deux heures et quart ; non dix minutes encore. Ils ne sont que sur le chemin du retour du déjeuner. Oui, mais la secrétaire $\mathrm{m}$ 'a dit « en début d'après-midi ». Après, ils rentrent en réunion. Donc, entre $14 \mathrm{~h}$ 20 et $14 \mathrm{~h}$ 23, j'ai une chance qu'il passe à son bureau. Il y a deux mois, il m'a dit : « Si je ne rappelle pas, vous me rappelez, il faut qu'on se décide vite sur ce coup. » Voilà un coup de fil qui de mon côté mûrit depuis deux mois.

Au moment où il est mûr, les choses ne vont pas sans un peu de trac. Un peu de Debussy, pour patienter. Se calmer avant les trois coups.

« Allô oui, il a laissé un message pour vous. Il a été obligé d'annuler tous ses rendezvous pour quinze jours. Je vous propose le 3 , oui, le 3 du mois prochain, à $10 \mathrm{~h} 30$. Vous me redonnez votre téléphone en cas d'empêchement. Je sais qu'il l'a, mais ça lui évitera de le rechercher. Bon après-midi ! » Et quand je raccroche, dans le silence éclate, il me semble, la tyrannique vieille rengaine de Bécaud, scandée au tambour: Tsan, Tata- ta-tsan, Ta-ta-ta-tsan ...

Et mainnntenannt,

Que vais-je faiiireu

De tout ce temmps ...» $(17 / 3 / 93)$

Convaincants, dérangeants, choquants parfois, ces propos restent cependant à la place qui leur est assignée par l'économie générale de l'enquête: celle de l'illustration. La narration des " témoins » interviewés est ainsi encadrée par la dissertation ${ }^{18}$ des gens légitimés à parler d'économie: journalistes, PDG, chercheurs. Aux victimes du chômage, les brèves confessions émouvantes - de quelques lignes à $1 / 4$ de page au maximum; aux élites l'apanage de la parole qui s'étire (1 page entière du Monde pour Jean Gandois, JeanBaptiste de Foucault, Alain Lebaube ou Michel Noblecourt), déroulant la prospective, le bilan, la conjecture, la synthèse.

Certes, il arrive que "Roberto » " Marie-Christine », « André », « Antoine » et les autres tirent de leur expérience quelques idées générales. Mais cela reste l'exception toujours à l'état d'ébauche, de l'ordre du cri réactif plus que de la réflexion véritable.

- « Pour Marie-Christine «c'est certain qu'on est très vite au bout du rouleau ». « Les suicides des chômeurs, il faut les comprendre. Je suis sûre qu'ils les cachent pour ne pas affoler les gens ». Elle raconte qu'elle éteint tout de suite la télévision « quand les hommes politiques parlent du chômage » : «La droite, la gauche, ils jouent au ping-pong sur notre dos ». Et quand elle voit des images de Sarajevo, elle se dit: « Peut-être que je suis égoïste mais je trouve qu'on devrait d'abord voir la misère en France, au coin de nos rues. On pourrait imaginer de faire le tri entre les Français vraiment français et les autres pour aider les Français au maximum. Il y a des choses que je ne comprends pas: on nous dit qu'on est en crise, qu'il n'y a plus d'argent, et on décide de supprimer la dette de l'Algérie, comme ça ». ». 
L'émotion, ou l'indignation passagère que peut engendrer la lecture de la page 19 du 11 mars 1993, celle du 17 mars ou celle des chroniques de J. P. Dautun ne résistent d'ailleurs pas au fatalisme qui imprègne l'ensemble du dossier. Le titre général de l'enquête - « Interminable chômage " - est révélateur, jusque dans son ambiguïté : chômage « interminable » à l'aune individuelle (les chômeurs " de longue durée ») autant que collective (une crise qui dure depuis 20 ans).

Quant à l'article liminaire, celui qui lance le dossier et donne le ton, il plante un décor totalement catastrophiste. Rédigé par un journaliste qui est en même temps un expert ${ }^{19}$, il se développe comme un article d'anticipation économique, sur le modèle du « scénario » qui permet aux journalistes, aux sociologues, aux démographes ou aux économètres de se transformer pour quelques instants en réalisateurs de «films catastrophes ${ }^{20}$. Il n'est pas non plus sans évoquer certains de ces jeux électroniques où le héros, à bout d'essais et de pistes infructueuses, finit par exploser dans un grand « crash » final :

«Quand on compte 3 millions de demandeurs d'emploi et que la société française se trouve dès lors menacée d'implosion, rien ne résiste.

Pour autant, et les propos de campagne électorale en témoignent, on peut se demander si au-delà du constat d'impuissance, les différents partis ont pris l'exacte mesure de ce qui était en train de se jouer, maintenant. Personne, parmi eux, n'aborde publiquement le scénario catastrophe qui hante beaucoup d'esprits actuellement, cette sorte de «big bang " social que certains prédisent avec effroi, des dirigeants d'entreprise aux observateurs les plus inquiets » / .../

«Au-delà, c'est l'implosion du corps social» / .../

« Par pans, l'alignement sur le « moins disant » provoquerait la destruction du tissu social et au cours des prochaines années, nous serions entraînés dans une spirale mortelle ».

Vocabulaire et images de la spirale, de l'accélération, de l'« effroi », de 1'« abîme ", jusqu'au rythme des phrases qui concourent dans cet article à produire le sentiment d'un « fatum » économique qui broie les hommes et les submerge.

«Derrière, le pire s'annonce, où tous les effets d'une économie mondialisée s'additionnent pour créer un enchaînement vertigineux et désormais incontrôlable. A tel point que la fameuse théorie du chaos, selon laquelle la propagation de l'onde due à un battement d'aile de papillon en Chine peut provoquer une tornade en Europe, ne reste pas seulement une belle image. Elle est en passe de devenir réalité».

Désignants de processus ("suppressions ", " croissance », etc), verbes pronominaux («s'accroître ») ou intransitifs (« sévir ») dessinent un univers sans responsabilité ni initiative humaine, où la «mondialisation» de l'économie et la «concurrence » occupent la place de l'antique « moira ».

«Alors que les suppressions d'emploi s'accélèrent (133 000 en 1992, après 92800 en 1991), il n'y a pas de raison d'espérer. Cette fois, la croissance est encore plus faible qu'en 1984-1985, quand les restructurations sévissaient à grande échelle ... A la différence du passé, aussi, la faiblesse de l'inflation n'accorde plus de marges de manoeuvre, au moment où les gains de productivité s'accroissent diaboliquement ${ }^{21}$. Dans ces conditions, dans un contexte de guerre économique, le chômage ne peut qu'augmenter, sans retenue $»^{22}$.

Les acteurs, les bénéficiaires, les motifs des "suppressions d'emploi », des « restructurations » et des " gains de productivité » ne sont pas explicités. La perception dominante de l'article s'apparente à celle du Soir 3 que nous avons analysé précédemment: perception nationaliste et " ensembliste ", où " l'Etat » représente « 
l'intérêt national " et où les "chefs d'entreprise " représentent les intérêts des entreprises, même si les uns et les autres peuvent diverger : intérêt national dont la logique est dépassée pour les chefs d'entreprise. Tandis que les pouvoirs publics tentent de protéger l'homogénéité du corps social ${ }^{23}$ avec d'ailleurs de plus en plus de difficultés ... les décisions de pure gestion, prises par les grands groupes aussi bien que par les PME, ne cessent de ruiner leurs efforts ».

Chacun fait ainsi ce qu'il peut: l'Etat - qui ne semble traversé par aucune contradiction gère au mieux le social et les « chefs d'entreprise $»^{24}$ gèrent au mieux les entreprises. Si tout va mal néanmoins, c'est la faute à la « mondialisation de l'économie » dont au bout du compte, tout le monde est victime :

"A l'instar de ce qui se passe aux Etats-Unis, la dualisation de la société pourrait s'installer durablement, et les ghettos avec elle, une partie de la population étant rejetée du coté du tiers ou du quart-monde, tandis que l'autre, mue par des réflexes sécuritaires, s'enfermerait dans sa richesse ».

La suite de l'enquête est à l'unisson. Des articles sur les théories économiques (13 mars), sur l'action des élus locaux (18 mars), sur la situation des autres pays européens (19 mars) ou sur les propositions politiques (20 mars), se dégage un sentiment d'impuissance souvent ramassé dans la « chute » :

- « La science économique a-t-elle fait pour autant les mêmes progrès que la médecine dans la connaissance des maladies? On peut, malheureusement, en douter ». (Dernière phrase de « Le blues des économistes devant les files d'attente ", 13 mars 1993).

- «Ce n'est pas le découragement qui guette aujourd'hui les acteurs économiques de Vierzon. Mais comment dire? Peut-être le sentiment que le déclic déterminant ne peut venir que d'un niveau qui les dépasse ». (Dernière phrase de "Le tonneau des Danaïdes ${ }^{25}$, 18 mars 1993).

Seuls les entretiens avec Jean Gandois, PDG de Péchiney (9/3/93) et avec Jean-Baptiste de Foucault, commissaire au plan (16/3/93) rompent avec la morosité de l'enquête. Mais comme dans Soir 3 , cette parole est celle des privilégiés de la carrière et des études ${ }^{26}$. Face à un journaliste qui n'est pas un contradicteur, ils lancent des pistes et émettent des voeux, aussitôt modulés et nuancés. Les formules sont générales, parfois même abstraites, se situant au niveau des principes plutôt que de l'action concrète.

C'est pourquoi l'interview de Jean-Baptiste de Foucault est aussi stimulant que décourageant. C'est un discours de futurs, de souhaits et de désirs qui se déroule au seuil de l'utopie, dessinant des avenirs de solidarités et de contrats, loin des rapports de force et des conflits d'intérêt actuels :

- « C'est précisément parce que l'organisation ou l'expression des exclus ne va pas de soi qu'elle doit être soutenue. Le fait que les cadres soient particulièrement touchés par les licenciements actuels peut d'ailleurs favoriser cette évolution. Ainsi la proposition d'un chèque syndical ou associatif qui serait versé à tout demandeur d'emploi de plus de six mois pour qu'il bénéficie d'une incitation forte à aller dans les structures d'accueil de son choix, et aussi pour que des structures d'accueil adaptées se créent mériterait d'être expérimentée dans quelques régions ».

- « Deuxième condition: il faut qu'une coopération s'instaure entre exclus et nonexclus. On ne sort pas quelqu'un de l'exclusion sans sa participation, et l'on ne peut pas sortir tout seul non plus de l'exclusion: il faut qu'un autre donne une chance.

- « Dans l'entreprise aussi, la résolution des problèmes de justice est un facteur d'efficacité, comme l'a montré la contribution de M. Praderie au colloque précité. Nous sommes sortis d'une vision naïve de l'égalité, ne tombons pas dans une vision 
simpliste de l'inégalité, condition obligée de performances, et retrouvons une certaine conception de la justice. C'est ainsi que nous pourrons aborder et traiter simultanément et correctement la double question de l'exclusion et de l'inégalité».

" Contractualisation ", « justice », « égalité ", « lutte contre l'exclusion », « aide », quel lecteur du Monde un tant soi peu humaniste n'acquiescerait à ces objectifs? Mais comment passer du monde de violences sociales et de souffrances décrit par Roberto ou MarieChristine dans l'article du 17 mars à ces lendemains d'intelligence et de compréhension? Certes Jean Gandois évoque " notre hypocrisie " et " notre niveau de vie " mais le réfèrent de ce «nous» demeure indéterminé :

«C'est un peu le problème de nos hypocrisies. Tout le monde veut en finir avec la misère horrible du tiers-monde. Tout le monde est pour la liberté des peuples opprimés. Mais quels sacrifices sommes-nous prêts à accepter sur notre niveau de vie? C'est le vrai problème du partage des revenus. Nous voulons être solidaires mais nous ne voulons pas payer les notes de la solidarité. Ayons le courage de notre hypocrisie ".

« Nous » global des sociétés dites «riches» face aux sociétés dites "pauvres »? Et où se situe lui-même Jean Gandois dans le "partage des revenus»? La question ne sera pas posée et l'interview se termine sur la vision irénique d'un futur grand " pacte social » succédant enfin à « un lourd passé de lutte des classes ».

Dans le Soir 3 du 1er juin 1993 consacré à «la crise allemande» et dans l'enquête du Monde du 7 au 20 mars 1993, nous voyons ainsi à l'oeuvre deux modalités d'amortissement du social. L'une repose sur l'adéquation totale du discours journalistique et du discours des " élites » et l'éviction de la parole des « dominés ». L'autre, plus subtile, et de ce fait plus difficile à saisir, enserre - on pourrait presque dire encercle - l'expression des souffrances et des injustices dans un ensemble d'analyses fatalistes et de propositions " morales " à long terme qui en désamorcent la charge de révolte ${ }^{27}$.

Au terme de ces deux études de cas, nous voudrions non point conclure, ni généraliser, car cela serait prématuré ${ }^{28}$ mais seulement resituer notre questionnement dans un cadre plus large.

Notre propos en effet n'est pas d'étudier les médias pour les médias mais de les étudier en tant qu'ils participent du politique et en tant que le politique passe par eux. Dans cette optique, il nous semble que la question de la parole et de ses modalités d'appropriation au sein des médias est essentielle pour la compréhension de l'organisation des pouvoirs et des influences dans notre société. Cette question n'est bien sûr pas nouvelle, comme il n'est pas nouveau que des élites sociologiques aient le monopole ou le quasi-monopole de l'expression politique. Mais si l'« inédit » est un critère essentiel pour qu'un sujet figure à la « une » des journaux, permanence et récurrence méritent tout autant l'attention du chercheur. C'est en ce sens que la comparaison entre la façon dont les députés du " peuple » disent la misère et l'exclusion au fil des assemblées républicaines et la façon dont les experts l'expriment aujourd'hui dans les médias, pourrait se révéler intéressante pour comprendre l'évolution de la démocratie moderne. " La politique est en effet, en grande partie, une lutte pour savoir qui a le droit de parler publiquement et au nom de qui. Elle est lutte pour la prise de parole et art de parler pour les groupes $»^{29}$. 


\section{BIBLIOGRAPHIE}

Authier-Revuz, J., (1982), « Hétérogénéité montrée et hétérogénéité constitutive : éléments pour une approche de l'autre dans le discours ", DRLA V n² 26, pp. 91-151.

Authier-Revuz, J., (1984), « Hétérogénéité(s) énonciative(s) », Langages n 73, pp. 98-111.

Authier-Revuz, J., (1985), «La représentation de la parole dans un débat radiophonique: figures de dialogue et de dialogisme ", Languefrançaise $\mathrm{n}^{\circ}$ 65, pp. 92-102.

Bakhtine, M., Volochinov, V.N., (1929), Le marxisme et la philosophie du langage: essai d'application de la méthode sociologique en linguistique, Paris, Editions de Minuit (trad. française 1977).

Bonnafous, S., (1991), L'immigration prise aux mots, Editions Kimé.

Champagne, P., (1990), Faire l'opinion, Editions de Minuit, Paris.

Mots 37, (décembre 1993), Rhétorique du journalisme politique, Presses de la FNSP, Paris.

Padioleau, J.-G., (1976), « Systèmes d'interaction et rhétoriques journalistiques » in Sociologie du travail $n^{\circ} 3$

Wallraff, G., (1986), Tête de turc, Ed. de la Découverte, Paris.

\section{NOTES}

1. « La presse libre et la démocratie souffrent des mêmes maux » conclut Jeanclaude Guillebaud dans un article intitulé « Les médias contre la démocratie » (Esprit, mars/avril 1993).

2. cf par exemple Lits et alii, M., 1993, La peur, la mort et les médias, Editions Vie Ouvrière, Bruxelles.

3. L'opposition habituellement opérée entre courant empirique et courant critique, très bien résumée par Jean-Louis Missika et Dominique Wolton dans La Folle du logis (Gallimard, Paris, 1983), mériterait d'être aujourd'hui révisée à la lumière des travaux " critiques " des dix dernières années. Souvent l'oeuvre de journalistes dits " alternatifs ", ils ont quitté les hauteurs philosophiques des Adorno, des Horkheimer ou Habermas pour examiner au plus près les conditions de production, d'énonciation et de circulation des médias contemporains.

4. Citons en particulier Leblanc, G., 1987, 13 -20h, Le monde en Suspens, Hitzeroth, Colon, M., 1992, Attention médias, Editions Epo, Bruxelles et Freund A., 1991, Journalisme et mésinformation, La Pensée Sauvage, Paris, sans oublier les très nombreux articles du Monde Diplomatique sur les médias, et en particulier ceux d'Ignacio Ramonet.

5. Notre analyse repose sur un travail préalable de transcription du JT que l'espace restreint d'un article ne nous permet malheureusement pas de reproduire.

6. Padioleau, .-G., 1976, « Systèmes d'interaction et rhétoriques journalistiques » in Sociologie du travail $\mathrm{n}^{\circ}$ 3, p. 256-282.

7. op. cit. p. 7 sq.

8. Authier-Revuz, J., 1982, « Hétérogénéité montrée et hétérogénéité constitutive : éléments pour une approche de l'autre dans le discours ", DRLA V n 26, pp. 91-151.

(1984) - « Hétérogénéité(s) énonciative(s) », Langages n 73, pp. 98-111.

(1985) - «La représentation de la parole dans un débat radiophonique: figures de dialogue et de 
dialogisme ", Languefrançaise $\mathrm{n}^{\circ} 65$, pp. 92-102.

Bakhtine, M. Volochinov, V.N. (1929) - Le marxisme et la philosophie du langage: essai d'application de la méthode sociologique en linguistique, Paris, Editions de Minuit (trad. française 1977)..

9. Champagne, P., 1990, Faire l'opinion, Editions de Minuit, Paris.

10. La seule personne sollicitée à propos des violences parfois meurtrières dont sont victimes les Turcs en Allemagne, se trouve donc être le Directeur du Centre de Documentation Juive de Vienne, internationalement connu pour avoir consacré sa vie à pourchasser les nazis réfugiés à l'étranger. Aucun représentant de la communauté turque n'est interrogé.

11. D'après Audiences-médias n 36, d'octobre 1992, « le téléspectateur type de Soir 3 est un homme âgé d'au moins 35 ans, plutôt citadin et de catégorie socio-professionnelle supérieure.

12. Les textes entre parenthèses sont de nous.

13. cf. in Gérard Leblanc, op. cit. : "Désignant en toutes choses l'instabilité du monde, l'information télévisée renforce la croyance qu'il est stable (ou devrait l'être ou pourrait l'être) », p. 16.

14. Tête de turc, Ed. de la Découverte, Paris, 1986.

15. Bonnafous, S., 1991, L'immigration prise aux mots, Editions Kimé, p. 46 à 100.

16. In Mots 37, Rhétorique du journalisme politique, Presses de la FNSP, Paris, décembre 1993.

17. Sur la formalisation très relative du travail journalistique, cf. Ruellan, D., 1993, Le professionnalisme du flou, identités et savoir-faire des journalistes français, PUG, Grenoble, (p. $132 \mathrm{sq})$.

18. La catégorisation proposée par Yves de la Haye dans Journalisme, mode d'emploi, (PUG, La Pensée Sauvage, 1983) se révèle ici tout à fait pertinente. Elle est présentée par Erik Neveu dans l'article de Mots 37 déjà mentionné.

19. Le 10 mars 1993, une publicité de la page 23 signale la parution d'un livre d'Alain Lebaube, intitulé « Social: par ici la sortie! » (Le Monde éditions).

20. Un des exemples les plus célèbres de ces dernières années fut l'émission intitulée « La guerre en face ", préparée conjointement par Le Point, Le Seuil et FR3 et présentée sur FR3 par Yves Montand le 18 avril 1985. L'intégralité du texte fut publié dans Le Point sous le titre "Scénario pour un cauchemar ». Rédigé par Laurent Joffrin, du journal Libération, à partir d'un ouvrage du général Etienne Copel, le scénario prévoyait l'invasion de l'Europe de l'Ouest par les forces conventionnelles de l'URSS.

21. On notera la « diabolisation» des gains de productivité qui contraste fortement avec la vision des experts du Soir 3 du 1er juin 1993.

22. Les soulignements en gras sont de nous.

23. Présupposé: le corps social est " homogène ». Comment faut-il l'entendre ? Culturellement, financièrement, idéologiquement?

24. Silence aussi dans cet article sur les conflits d'intérêt entre actionnaires et P.-D.G, pourtant à l'origine de plusieurs limogeages de ces derniers aux Etats-Unis en 1993.

25. On notera au passage la contradiction entre le chapeau qui évoque « une rébellion contre la fatalité : l'histoire de Vierzon » et le titre qui puise dans la mythologie un des exemples les plus célèbres d'impuissance humaine et de châtiment divin.

26. Les curriculums de MM. Gandois et de Foucault sont typiques de l'élite politique et économique française : Ancien élève de l'Ecole polytechnique et ingénieur des Ponts et Chaussées, Jean Gandois a fait toute sa carrière dans l'industrie: directeur général de Sacilor, P.D.G de Sollac puis P.-D.G, de 1979 à 1982, de Rhône-Poulenc, il est, depuis 1986, P .-D.G de Pechiney et, depuis 1987, président du groupe Cochrill Sambre. Militant pour une organisation plus qualifiante du travail, Jean Gandois a présidé, dans le cadre de la préparation du XIe Plan, la commission « Compétitivité française». (9 mars 1993, p. 2).

"Né en 1943, ancien élève de l'E.N.A., inspecteur des finances, Jean-Baptiste de Foucault a été conseiller au cabinet de Jacques Delors, alors ministre de l'économie (1982-1984), dont il est resté proche puisqu'il anime le club Echanges et Projets, avant de rejoindre le Commissariat du Plan 
comme second (de 1988 à 1992) puis comme patron. Il est notamment l'auteur de La Fin du socialcolbertisme (Be1fond, 1988). (16 mars 1993, p. 2). Alain Labaube, perçoit d'ailleurs le paradoxe qu'il $\mathrm{y}$ a à ne solliciter de propositions sur le chômage ou le partage du travail que de ceux qui ont tout - jusqu'à l'excès de travail sans doute. Dans l'article du 10 mars consacré au « choc des 3 millions ", il fait état d'une enquête sur l'emploi commandée par l'Institut de l'Entreprise. Et de prévenir lui-même: «Face à trois millions de chômeurs, une telle initiative paraîtra dérisoire. Certains la jugeront inutile ou, à la lecture de quelques noms - Jean Gandois, président de Pechiney, Michel Bon, ancien P.-D.G de Carrefour, Francis Mer, président d'Usinor-Sacilor, parleront d'hypocrisie. Les « licencieurs » ne peuvent pas être les conseilleurs. Il ne faut pourtant pas sous-estimer la valeur de ce signe qui, parmi beaucoup d'autres, tend à montrer que les choses changent et qu'une prise de conscience est sans doute en train de se produire ».

27. Une troisième forme $d^{\prime}$ " amortissement » du social réside à notre avis dans la rhétorique de l'expertise professionnelle. Présente dans le domaine du logement, de la santé, de la ville, du social, de l'éducation, etc..., elle justifierait une étude spécifique des rapports d'évaluation des politiques publiques.

28. Un numéro de la revue Mots (Presses de la Fondation Nationale des Sciences Politiques) est en préparation sur le discours des exclus de l'emploi et/ou du logement. Il s'interrogera sur la parole « populaire » saisie par Pierre Bourdieu et son équipe dans La misère du monde (Paris, Seuil, 1993) et proposera une première approche, en terme de locuteurs et d'énonciateurs, des journaux de SDF récemment apparus en France.

29. P. Champagne, op. cit. p. 252.

\section{RÉSUMÉS}

L'analyse de deux corpus de presse, un journal télévisé de France 3 et une enquête du Monde, tous deux datés de 1993, permet de mettre à jour deux modalités du discours médiatique sur la crise et le chômage. L'une repose sur l'adéquation totale du discours journalistique et du discours des « élites ». L'autre utilise le témoignage des " exclus» comme une simple illustration d'un discours d'experts qui oscille entre le fatalisme et le moralisme. L'étude de la distribution et de la hiérarchisation de la parole manifeste une concordance entre ces deux modes de représentation d'un problème social: les procédures de globalisation et le jeu des formes énonciatives contribuent à un «amortissement du social» et des charges affectives qu'il pourrait susciter. Ces études de cas s'inscrivent dans une interrogation plus générale sur la structuration de la parole dans l'espace public contemporain.

The author analyses two press corpuses, news bulletin on France 3 and a survey in Le Monde both in the year 1993. She brings into light two methods used in mediatic speeches about crisis and unemployment. The first one assumes that speeches from political men and experts are true and sticks to them. The second one gets the unemployed to reports about their situation but considers their statements as providing examples to the analyses given by the experts who are either complaining about fatality or suggesting a moral effort. If we consider the place and importance given to the different speeches it appears that whatever statements they make about this social problem, the result is the same. The process of generalization and the play of enunciation lead to a cover-up of social problems and the affects they might bring about. These 
analyses are part of a more general query about the role played by con temporary medias in the structure of speeches.

INDEX

Mots-clés : crise, média, économie

Keywords : crisis, media, economy

\section{AUTEUR}

\section{SIMONE BONNAFOUS}

Simone Bonnafous, Université Paris XII- Val de Marne et Laboratoire de lexicométrie des textes politiques de l'E.N.S. de St-Cloud/Fontenay 